\section{Kinder- und Jugendpsychiatrie für alle}

Prof. Dr. Dr. Helmut Remschmidt und seine Mitautoren zeichnen in der sechsten überarbeiteten Auflage des Buches "Kinder- und Jugendpsychiatrie“ ein umfassendes wissenschaftlich fundiertes, praxisorientiertes Bild der Diagnostik und Behandlung psychischer Störungen im Kindes- und Jugendalter auf.

Helmut Remschmidt, seit 2006 emeritiert, war zuletzt langjähriger Lehrstuhlinhaber für Kinder- und Jugendpsychiatrie und -psychotherapie der PhilippsUniversität Marburg. Sein wissenschaftliches Interesse galt den neurobiologischen/genetischen Grundlagen der Kinder- und jugendpsychiatrischen Forschung. Er baute ein regionales Versorgungssystem für psychisch kranke Kinder auf. Die 23 Koautoren sind langjährig klinisch tätige Fachärzte, Psychologen und Unterrichtende.

Die Neuauflage des handlichen $\mathrm{Bu}$ ches ist in sechs Teile gegliedert, die folund Entwicklungspsychopathologie, kinder- und jugendpsychiatrische Diagnostik, psychische Störungen und Erkrankungen bei Kindern und Jugendlichen, Therapie und Rehabilitation, gerichtliche Aufgaben und Organisations-/ Strukturfragen der Kinder- und Jugendpsychiatrie sowie Literatur zur Kinderund Jugendpsychiatrie und ihren Randgebieten.

Die Autoren dieses Buches richten sich an einen interdisziplinären Leserkreis. Die gut verständlichen Texte werden durch zusammenfassende Tabellen und Therapiekonzepte, schematische Darstellungen der Pathopsychologie und Patientenbeispiele aufgelockert. Ansonsten bleibt der Aufbau durchgängig klar strukturiert und übersichtlich. Bei den meisten Patientenbeispielen werden Verlauf und Therapie mit angegeben. Die Einordnung der Krankheitsbilder im gende Themen behandeln: Entwicklung

ICD10 verschafft einen Einblick in die diagnostischen Schwierigkeiten. Der Anspruch des empirisch, evidenzbasierten Konzepts ist hier gelungen. Gerade der bewusste Schwerpunkt auf die Ätiopathologie motiviert den fachfremden Leser, die Grenzen zwischen somatischer und psychiatrischer Medizin weiter aufzuweichen. Die gelungene $\mathrm{Mi}$ schung aus Faktenwissen und Anleitung im Umgang mit Patienten und Eltern verdeutlicht das Spannungsfeld der Kinder- und Jugendpsychiatrie. Insgesamt ist das Buch für die Einführung in die Kinder- und Jugendpsychiatrie mit ihren vielen Facetten und Anforderungen bestens geeignet.

Dr. Caroline Maxton

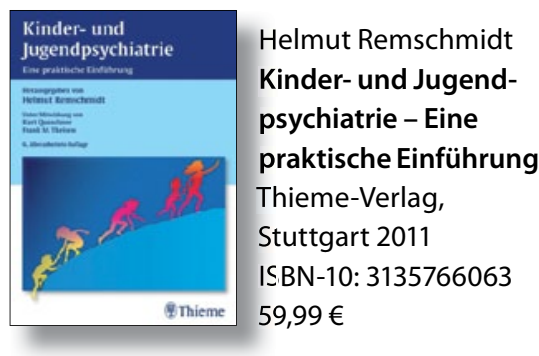

menten in der Lage sind, ein kognitives Enhancement zu betreiben, wird hier mit Literaturangaben widerlegt. Das Wissen über die Nebenwirkungen dieser Medikamente ist dem Autor leider weniger geläufig. So verbreitet er noch die Information, dass Methylphenidat $\mathrm{zu}$ Wachstumsminderung führt - dies entspricht nicht der aktuellen Studienlage. Seriöse Literaturangaben vermisst der Leser auch, wenn der Autor von häufigem Auftreten von Herzrhythmusstörungen unter Methylphenidat schreibt. Trotzdem ist das Buch zu empfehlen für alle, die sich mit Intelligenz, der Definition und Messung von Intelligenz sowie insbesondere mit den Möglichkeiten der Beeinflussung von Intelligenz beschäftigen. Dr. Kirsten Stollhoff

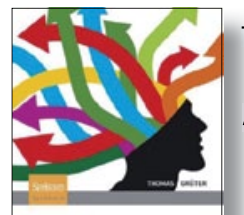

Thomas Grüter Klüger als Wir Auf dem Weg zur Hyperintelligenz Spektrum Akademischer KLÜGER

ALS WIR? Verlag, Heidelberg 2011 ISBN-10: 3827426480 reich, sich über Intelligenz $\mathrm{zu}$ informie-

nach Machterhalt. Gerade in einer Zeit, in der in den Medien immer wieder "Neuroenhancement" vorgeworfen wird, wenn neurobiologische Krankheiten wie ADHS behandelt werden, ist es hilfren. Die These, dass wir mit Medikatem Auftreten von psychischen Störun- 